\title{
LA GENTE MALA DEL NORTE: MIRADA TRANSGENERACIONAL DE ESTEREOTIPO NORTINO EN UNA MUESTRA DE MAGALLÁNICOS
}

ERIC MOLINET \& CLAUDIA ESTRADA*

\begin{abstract}
RESUMEN
El presente estudio se interesa en el fenómeno del estereotipo de las personas pertenecientes a regiones diferentes a Magallanes, denominadas "la gente mala del norte". Una muestra de 81 habitantes de la ciudad de Punta Arenas participaron voluntariamente en este estudio siendo seleccionados por muestreo no probabilístico por cuotas. Contestaron a dos instrumentos de evaluación de estereotipos, uno por diferencial semántico y el otro por generación de adjetivos. Mediante dichas técnicas se evaluaron las categorías sociales: magallánicos (auto-estereotipo), chilotes, viñamarinos, nortinos y santiaguinos. Los resultados indican que el grupo evaluado menos positivamente es el que corresponde al de los santiaguinos los que presenten un estereotipo ambivalente. La diferencia entre grupos de edad indica que los jóvenes evalúan negativamente a este grupo mientras que los adultos se sitúan en la neutralidad. Los resultados se discuten en torno a la base empírica del mito de la "maldad del nortino" y sus consecuencias para la convivencia regional y nacional.
\end{abstract}

PALABRAS CLAVE: estereotipo, identidad, categorías sociales.

\section{EVIL PEOPLE OF THE NORTH: A TRANSGENERATIONAL GLANCE TO THE NORTHERN STEREOTYPE IN A MAGELLANIC SAMPLE}

\section{ABSTRACT}

This study examines the phenomenon of stereotyping belonging to different regions other than Magellanes, called "the evil people of the north". A sample of 81 inhabitants of the city of Punta Arenas participated voluntarily in this study being selected by non-probabilistic sampling by quotas. They responded to two instruments to assess stereotypes, one semantic differentiales, and the other by generating adjectives. Using these techniques were evaluated social categories: Magellan (auto-stereotype), people from Chiloe, native of Viña del Mar, people from de north and from Santiago city. The results indicate that the group less positively evaluated is that which corresponds to that of Santiago showing the ambivalent stereotype. The difference between age groups indicates that young people assess negatively to this group while the adults are in neutral. The results are discussed regarding the empirical basis of the myth of the "the evil people of the north" and its implications for regional and national coexistence.

KEY WORDS: stereotype, social identity, social categorization.

*Universidad de Magallanes, Av. Bulnes 01855, Punta Arenas. Chile. claudia.estrada@umag.cl. 


\section{INTRODUCCIÓN}

Por mucho tiempo en la región de Magallanes se tiene la impresión de que existe una mala reputación sobre los "nortinos" y que estos son vistos como personas que mal influencian y/o mal actúan. Es probable que esta visión un tanto negativa hacia el nortino pudiese tener su génesis a comienzo del siglo XX, en esta época el estado chileno cae en un olvido con el extremo sur. Este sentimiento de exclusión con un proyecto nación, provoca un sentimiento de abandono, de no reconocimiento, $y$ ante este escenario, se agrupan con mayor fuerza las aspectos vinculados al "nosotros". Esta identidad patagónica surgida desde la negación configura una identidad colectiva que bordea dos espacios, el nosotros magallánicos y el nosotros nacional (Facchinetti et. al., 2000).

El rechazo por el norte en un comienzo se dirige principalmente hacia el centralismo excesivo, y rescata el autogobierno o la autogestión como una alternativa para el futuro, sin renunciar a la unitariedad del Estado (Martinic 1992).

Cabe señalar que para muchos en la región de Magallanes son considéranos nortinos no sólo aquellos que viven al norte de la región Metropolitana sino que todas las personas que habitan desde la primera a la décima región.

Desde mucho tiempo en Magallanes ha existido una gran inmigración de personas provenientes del norte, la mayoría de estas temporalmente, lo que ha hecho que constantemente exista una interrelación que ha forjado de una u otra manera una percepción en donde se ha escuchado más de alguna vez esa ya mítica frase "gente mala del norte".

La magnitud de esta situación abarca a toda la región de Magallanes, ya que el concepto de que los "nortinos" afectan el buen vivir de la región es transversal, o por lo menos eso supone. La forma en que esto se manifiesta anecdóticamente con expresiones que son visibles tanto en el diario local como en las redes sociales: robo en Porvenir, se sospecha de afuerinos, o la gente del norte quiere hacerse rico a costa nuestra. Incluso existe una película estrenada el 2011 homónima que relata los hechos acaecidos en torno a un asesinato ocurrido en 1923, y en que se relata la forma como este prejuicio se manifiesta en un hecho concreto.

Si a lo anteriormente planteado agregamos que la región de Magallanes tiene una especificidad histórica y que está además geográficamente aislada, separada sin vuelta del resto del territorio nacional, toma más fuerza esa identidad regional con anhelos de autogobierno, de autogestión, etc. (Martinic, 1992). Este proceso de construcción de la identidad magallánica, es complementado con otros aspectos que movilizan ese mismo eje.

A partir de estos acontecimientos, probablemente se comenzó a generar esa idea de tenacidad, de pioneros, que no sólo se es capaz de levantarse ante la "nada", sino también hacia la exclusión de un sentir país. Este doble juego de inclusión-exclusión del proyecto país comienza a ejercer una diferenciación en la conformación de la identidad magallánica (Cruz, 2000).

Es interesante desde el punto de vista psicosocial el cómo son percibidos los "nortinos" en Magallanes, si detrás de esa supuesta "mala percepción" se esconde más mitología que realidad. Además de conocer si existe alguna diferencia entre las generaciones de jóvenes y adultos magallánicos. Hacer visible los estereotipos existentes de los magallánicos hacia los habitantes del norte.

Desde que Lippmann en 1922 acuñara el término "estereotipo" para designar esas imágenes en nuestra cabeza, que median entre la realidad y la percepción que nosotros tenemos de ella, se han ofrecido distintas explicaciones teóricas sobre el concepto mismo del estereotipo y se han realizado cientos de trabajos sobre estereotipos ya sean raciales, de género, nacionales, religiosos, etc. (Krech, 1978).

Actualmente, existe cierto acuerdo en considerar los estereotipos como resultado de procesos de categorización, y referidos a aquellas creencias populares sobre los atributos que caracterizan a una categoría social y sobre los que existe un acuerdo sustancial (Mackie, 1973). Para Morales \& Moya (1996), un estereotipo consiste en un conjunto de creencias, compartidas, acerca de los atributos personales que poseen los miembros de un grupo. Los estereotipos provocan una simplificación o una orientación selectiva de nuestras percepciones que podrían conducir a distorsiones de la realidad objetiva. Estos han insistido en el papel fundamental jugado por este proceso en los estereotipos, dado que la percepción diferencial de distintos grupos sociales es imposible sin una previa categorización de las personas como pertenecientes a un grupo $\mathrm{u}$ 
otro (Klineberg, 1973)

No se pretende que todos los miembros de una categoría presenten todos los rasgos definitorios de la misma, y sean todos ejemplares iguales. Más bien, las categorías de personas se consideran como las típicas organizadas en torno a casos prototípicos (Secord \& Beckman, 1976). Así, las categorías sociales serían de cierta manera probabilísticas. En este sentido, los estereotipos serían realmente "predicciones probabilísticas" sobre las características que distinguen a unas categorías de personas de otras (McCauley, Stitt \& Segal, 1980).

Desde un enfoque cognitivo, los estereotipos se consideran como conceptos cognitivo-estructurales, referidos a un conjunto de expectativas sostenidas por un perceptor sobre los miembros de un grupo social (Hamilton, 1979). En este sentido, influirían, a modo de esquemas en el procesamiento de la información que recibe sobre personas o grupos y, en concreto, durante los procesos de codificación $e$ interpretación de la información. Aquí surgen los llamados autoestereotipos y los heteroetereotipos, los primeros el conjunto de creencias y percepciones sobre si mismo y el grupo del cual forma parte, y el segundo son las percepciones que tememos de los demás grupos (Morales et al. 2002).

Los estereotipos facilitan la identidad social del individuo, al ayudarle a definir su lugar en la sociedad (Tajfel, 1978). En efecto, la identidad social, ese conocimiento que tiene el individuo de pertenecer a ciertos grupos o categorías sociales, junto con el significado emocional y evaluativo resultante de tal pertenencia (Tajfel, 1972), no puede definirse más que a partir de la categorización social, que actúa segmentando el entorno social del individuo (Tajfel, 1984).

Según Hogg \& Abrams (1999) estos estereotipos sociales se deben a un proceso de influencia social que genera conformidad a las normas grupales. Así la teoría de identidad social plantea que los estereotipos son consensuales porque todos los miembros del grupo social se supone deben seguirlos con la finalidad de establecer justificaciones colectivas para el comportamiento intergrupal.

En ciertos momentos la teoría de la identidad social sugiere que los individuos están motivados para formar estereotipos positivos del endogrupo, y, en otros momentos, que los estereotipos del endogrupo reflejarán la posición del grupo en la sociedad, pudiendo ser negativa o positiva. Por ejemplo Hogg \& Abrams (1999), describen que existiría un interés establecido por preservar la superioridad evaluativa del endogrupo a toda costa, mientras que Hogg \& Turner (1987) plantean que la forma que tome el autoestereotipo dependerá de las relaciones entre los grupos. La teoría parece aceptar que los grupos con poco poder, internalizarán las normas de los poderosos, pero también predice que los grupos con escaso poder, desarrollarán sus propias normas para lograr distintividad social positiva. Las personas atribuirán rasgos a sí mismos y a los otros en forma tal que el status o rol que desempeñan sea justificado (Ross et. al. 1976).

En este sentido, poca duda cabe de que los estereotipos juegan asimismo un papel relevante en relación a la identidad regional o nacional de un pueblo. Al fin y al cabo, son estimaciones de carácter regional o nacional, y de hecho, en la literatura sobre estereotipos suele defenderse la existencia en ellos de un "fondo de verdad" que, por muy simplificado, exagerado, incompleto, etcétera, que sea, está probablemente cerca del carácter propio de un pueblo. Esto es singularmente cierto en aquellos rasgos que aparecen de manera regular tanto en los autoestereotipos como en los heteroestereotipos de un pueblo (Franzoi, 2007).

Son de gran importancia en las relaciones sociales los estereotipos, ya que estos son el primer paso para el prejuicio, concebidos como pensamientos, conductas o afectos negativos hacia ciertos grupos y sus integrantes. Se trata de una definición más amplia que la de estereotipo, pues no sólo incluye a las creencias o pensamientos que se tienen sobre los inmigrantes o personas de determinado lugar geográfico o de nacimiento, sino también a los afectos y conductas hacia ellos (Allport, 1977).

Actualmente también se señala que el prejuicio es una ideología que justifica la desigualdad entre los grupos, y esto se consigue no sólo con una imagen negativa de otros grupos, sino a veces transmitiendo una imagen positiva de ellos, aunque limitada a ciertas dimensiones (los miembros de ciertos grupos pueden ser vistos como "espontáneos", "afables", "divertidos", y aunque esto sea positivo, puede estar favoreciendo el mantenimiento de este grupo en una situación de inferioridad) (Worchel, 2004).

En todo caso el trabajo de investigación 
no pretende buscar las causas de esta situación, sino conocer si es que en realidad existe una mala percepción hacia los "nortinos", cómo está construida (contenido del estereotipo), cuál es su especificidad (grupo de "nortinos") y si esta se ve afectada por la diferencia generacional.

No se ha abordado mayormente esta temática y no existe mayor conocimiento empírico sobre los estereotipos de los magallánicos hacia los nortinos, estos se dan sólo a través de rumores o construcciones mitológicas que en la actualidad no se saben cuánto de verdad tienen estas creencias que los magallánicos tendrían una mala impresión de las personas del norte.

En la actualidad no se cuentan con trabajos que apunten a obtener conocimientos de cómo se percibe al nortino en la región de Magallanes, los intentos pudiesen haber existido, pero sólo quedó en eso. Además la percepción con relación a los "nortinos" no es vista como un problema, más bien existe la creencia popular que dice que los magallánicos creen que ciertas actitudes o actos no deseados provienen de personas del norte del país o influenciados por éstas. La creencia que las personas originarias del norte del país son vistas por los magallánicos como no gratas, que ejercen ciertas actitudes que no son deseadas por la comunidad.

Esta investigación dará a conocer ciertas tendencias de la impresión que tienen los adultos y jóvenes magallánicos con relación a las personas denominadas "nortinas". Además de ver si existen diferencias entre estos dos grupos de magallánicos con relación a esta visión. Lo cual podría ayudar a conocer cuánto de verdad y cuánto de mito existe en que los magallánicos tienen ciertos prejuicios hacia los "nortinos"

\section{MÉTODO}

\section{Participantes:}

La muestra, no probabilística por cuotas, estuvo constituida por 81 personas de la ciudad de Punta Arenas, auto-categorizados como magallánicos, dividida en dos grupos, el primero de jóvenes que representa al $49.5 \%(M=21,7 ; D . E=2,52)$ de la muestra total y el segundo de adultos con el $50.6 \%$ $(M=60,1 ; D . E=11,12)$. Según el género, el 40.7\% de los participantes de la encuesta fue de género masculino y un $59.3 \%$ femenino.
Los niveles de escolaridad de los participantes de la encuesta según grupo de edad: el $97.5 \%$ de los jóvenes se encuentran cursando "estudios superiores", en tanto que el 2.5\% restante presentaba "enseñanza media completa". En el caso del grupo de adultos la moda de escolaridad se ubicó en la categoría "enseñanza media incompleta". El $70.7 \%$ de los adultos encuestados se encuentra en los niveles de escolaridad que van desde "básica incompleta" hasta "media completa", el 26.9\% de los participantes de este grupo de edad tuvo instrucción en niveles de educación superior, que van desde estudios en institutos técnicos incompletas hasta estudios de postgrado. El 2.4\% no tuvo enseñanza en ningún establecimiento de educación formal.

\section{Diseño:}

Se utilizó un diseño no experimental transaccional correlacional de diferencia entre grupos.

\section{Instrumentos:}

Escala compuesta de dos partes. La primera corresponde a la evaluación de estereotipo mediante diferencial semántico (Osgood et al. 1976) con cinco parejas de adjetivo opuestos: bueno/malo, positivo/ negativo, no valioso/valioso, desagradable/agradable, atrayente/repulsivo, con los cuales se midieron las categorías de magallánicos, chilotes, viñamarinos, nortinos y santiaguinos. Esto con el fin de observar cómo los participantes de la investigación evalúan a estos diferentes grupos. Se utilizó una escala Osgood de 7 puntos. Los adjetivos fueron recogidos a partir de una serie de estímulos que fueron utilizados en otras investigaciones similares. Luego se le solicitó a un grupo de voluntarios de definieran los "antónimos", es decir, aquellos términos que en el lenguaje cotidiano son significados como contrarios. Esto permitió definir inicialmente las dimensiones, en la que no se consideró la dimensión bueno/menos bueno o malo/menos malo, como recomiendan sus creadores debido a que el pretest determinó que resultaban poco familiares para quienes respondían a las escalas. El siguiente paso fue la medición de la dimensión evaluativa de los términos (no se determinó ni la potencia ni la actividad) para asegurarse que eran considerados como contrarios (diferentes). Aunque se realizó un AFE de componentes principales, este arrojó la presencia de un factor principal que explica el 56,6\% de la varianza, lo que se interpreta 
en la dirección de la existencia de una dimensión (negativo-positivo) que es el factor principal de la escala propuesta.

Evaluación de estereotipo mediante producción de adjetivos (Allen \& Potaky, 1973): Los participantes debieron producir de manera espontánea una serie de adjetivos describiendo a los magallánicos, chilotes, viñamarinos, nortinos y santiaguinos, para posteriormente evaluar la valencia de esas características sobre una escala tipo Likert para cuantificar con puntajes que van de -3 que es el (extremo negativo) a 3 (extremo positivo).

Procedimiento:

Para el grupo de jóvenes se acudió a su

Tabla 1. Promedios y desviaciones estándar resultantes de la evaluación mediante diferencial semántico.

\begin{tabular}{|c|c|c|c|}
\hline Grupo & Media & D.E. & $M \neq 0$ \\
\hline \multicolumn{4}{|l|}{ Magallánico } \\
\hline Malo - Bueno & 2,31 & 0,82 & 0,0001 \\
\hline Negativo - Positivo & 1,65 & 1,38 & 0,0001 \\
\hline No valioso - Valioso & 2,35 & 1,02 & 0,0001 \\
\hline Desagradable - Agradable & 2,20 & 1,01 & 0,0001 \\
\hline Repulsivo - Atrayente & 1,61 & 1,44 & 0,0001 \\
\hline \multicolumn{4}{|l|}{ Chilote } \\
\hline Malo - Bueno & 2,05 & 1,26 & 0,0001 \\
\hline Negativo - Positivo & 1,74 & 1,41 & 0,0001 \\
\hline No valioso - Valioso & 2,15 & 1,28 & 0,0001 \\
\hline Desagradable - Agradable & 1,91 & 1,30 & 0,0001 \\
\hline Repulsivo - Atrayente & 1,46 & 1,49 & 0,0001 \\
\hline \multicolumn{4}{|l|}{ Viñamarino } \\
\hline Malo - Bueno & 0,98 & 1,41 & 0,0001 \\
\hline Negativo - Positivo & 1,28 & 1,27 & 0,0001 \\
\hline No valioso - Valioso & 1,34 & 1,36 & 0,0001 \\
\hline Desagradable - Agradable & 1,13 & 1,36 & 0,0001 \\
\hline Repulsivo - Atrayente & 1,26 & 1,35 & 0,0001 \\
\hline \multicolumn{4}{|l|}{ Nortino } \\
\hline Malo - Bueno & 0,53 & 1,77 & 0,008 \\
\hline Negativo - Positivo & 0,63 & 1,51 & 0,0001 \\
\hline No valioso - Valioso & 0,93 & 1,53 & 0,0001 \\
\hline Desagradable - Agradable & 0,58 & 1,47 & 0,001 \\
\hline Repulsivo - Atrayente & 0,38 & 1,52 & 0,029 \\
\hline \multicolumn{4}{|l|}{ Santiaguino } \\
\hline Malo - Bueno & 0,00 & 1,67 & 1,000 \\
\hline Negativo - Positivo & 0,11 & 1,59 & 0,529 \\
\hline No valioso - Valioso & 0,81 & 1,50 & 0,0001 \\
\hline Desagradable - Agradable & 0,33 & 1,57 & 0,067 \\
\hline Repulsivo - Atrayente & $-0,01$ & 1,61 & 0,945 \\
\hline
\end{tabular}

lugar de estudio en donde voluntariamente se les pidió que contestaran el instrumento. Posteriormente se entregó a cada participante un documento de participación consentida el cual debían leer y firmar si se encontraban de acuerdo con lo ahí establecido Se les explicó en qué consistían los cuestionarios y el objetivo general del estudio.

En el caso del grupo de adultos la aplicación del instrumento fue de manera personalizada, efectuándose principalmente en el lugar de trabajo de estos, además de domicilios y grupos de encuentros como juntas de vecinos. En el proceso de aplicación se realizó lectura completa del instrumento una vez concluido esta lectura se hizo entrega del documento de participación consentida el cual también se leyó en conjunto.

Una vez terminada esta etapa a ambos grupos de edad se les entregó información por escrito con los datos necesarios para poder realizar la petición de los resultados que se obtuviesen de la investigación, se aclararon dudas y se les agradeció por su participación.

\section{RESULTADOS}

Los análisis realizados sobre estereotipos mediante diferencial semántico, indican que los Magallánicos se ven a sí mismo de forma positiva en todas las características consultadas. En orden descendente de positividad encontramos: Bueno $(96,3 \%)$, valioso $(93,8 \%)$, agradable $(92,5 \%)$, positivo $(78,8 \%)$ y finalmente atrayente $(77,5 \%)$.

En el caso de los chilotes, También la descripción se adscribió a los polos positivos de las dimensiones. En orden descendente Bueno $(90,1 \%)$, valioso (85\%), agradable (81,3\%), positivo (80\%) y atrayente $(71,3 \%)$

En el caso de los viñamarinos el perfil característico fue similar a los grupos anteriores. Agradable (68,8\%), atrayentes (66,3\%), positivo y valioso ambos con un $65 \%$ y finalmente bueno $(60,5 \%)$

Los nortinos son descritos mayoritariamente como valiosos $(58,8 \%)$, seguido de buenos con un $53,1 \%$, positivo con un $52,5 \%$, agradable lo considera un $51,3 \%$ y en el caso del continuo atrayente/ repulsivo, un $44,3 \%$ considera atrayentes a los nortinos, mientras que un $55,7 \%$ no los considera como tal (un $20,3 \%$ los ve como repulsivos y un 
35.4\% se sitúa en la neutralidad)

Por último, la caracterización de los santiaguinos muestra un perfil diferente a las otras categorías sociales. Los participantes señalan que son valiosos $(53,8 \%)$, agradables $(46,3 \%)$ versus desagradable $(23,8 \%)$, bueno $(38,3 \%)$ versus malo (37\%), positivo (37,5\%) versus negativo $(32,5 \%)$, y atrayente (30\%) versus repulsivo $(28,8 \%)$.

Los promedios resultantes de cada característica y para cada grupo muestran que los promedios más altos, atribuibles a características positivas, son asignados particularmente a la categoría magallánico, siendo las categorías nortino y santiaguino, las que presentan menores promedios (Ver Tabla 1)

Al comparar la descripción de características según grupo de edad (jóvenes/adultos), con nivel educacional como covariante, se observa que existen diferencias en el uso de sólo tres características todas ellas y sólo al referirse al grupo Magallánicos. La primera, marginalmente significativa, se presenta al momento de calificar a los magallánicos como No valioso/Valioso, los jóvenes muestran un puntaje promedio menor $(M=2,10, D E=1,24)$, que el grupo de adultos $(M=2,60, D E=0,67), F_{(1,80)}=$ $3,13, p=0,08$. En otros términos, aunque ambos grupos señalan que los magallánicos se asocian al término "valioso", los adultos tienden a percibir esta característica como más extrema que los jóvenes.

La segunda diferencia que se observa es en la categoría desagradable/agradable, aquí también los jóvenes presentan un promedio menor $(M=2,05, D E=0,96)$, que los adultos $(M=2,35$,

Tabla 2. Frecuencia de características para los magallánicos

\begin{tabular}{lc}
\hline \multicolumn{1}{c}{ Característica } & \% de acuerdo \\
\hline Amigables & $85,2 \%$ \\
Trabajadores & $55,6 \%$ \\
Solidarios & $40,7 \%$ \\
Regionalistas & $37,0 \%$ \\
Alegres & $27,2 \%$ \\
Conservadores & $25,9 \%$ \\
Hogareños & $22,2 \%$ \\
Responsables & $14,8 \%$ \\
\hline
\end{tabular}

$D E=1,05), F_{(1,80)}=4,51, p=0,04$. En la última categoría en la que se observaron diferencias es la que corresponde a atrayente/repulsivo, aquí el grupo de jóvenes presenta nuevamente un puntaje promedio menor $(M=1.28, D E=1.56)$, con relación el grupo de adultos $(M=1.95, D E=1.24)$, indicando que los adultos consideran a los magallánicos como más atrayentes que los jóvenes, $F_{(1,80)}=5,48, p=$ 0,02 . En las demás categorías no se observaron diferencias significativas al momento de evaluar a los demás grupos.

Luego de levantar los adjetivos utilizando la lista de técnicas de generación espontánea, se realizó un análisis de contenido con vistas a reagrupar los adjetivos similares en categorías más amplias. Luego de este procedimiento, se escogieron aquellos que mostraron mayor representatividad.

El análisis de los datos recolectados mediante la técnica de generación espontánea de adjetivos permite conocer la configuración de adjetivos más típica según los participantes para cada categoría social evaluada. A continuación se presentan los resultados tanto del auto-estereotipo como de los estereotipos resultantes

Al observar las frecuencias de las características expresadas por los encuestados con relación a los Magallánicos se encontró que los adjetivos más comunes fueron "amigables" y "trabajadores" (Tabla 2).

Para el caso de los santiaguinos podemos observar que el mayor porcentaje se sitúa en la característica "agresivos" seguida por "delincuentes" y "extrovertidos" (ver Tabla 3).

Tabla 3. Frecuencia de características para los santiaguinos

\begin{tabular}{lc}
\hline \multicolumn{1}{c}{ Característica } & \% de acuerdo \\
\hline Agresivos & $40,74 \%$ \\
Delincuentes & $32,01 \%$ \\
Extrovertidos & $32,01 \%$ \\
Individualistas & $29,63 \%$ \\
Estresados & $28,40 \%$ \\
Trabajadores & $23,46 \%$ \\
\hline
\end{tabular}

Al evaluar la valencia asignada a cada estereotipo, observamos que los Magallánicos ( $M=$ 2.0, $D E=1.03)$ y chilotes $(M=2.1, D E=1.11)$ son evaluados de forma similar $t c_{(80)}:-1.13$, n.s., mientras que ambos son diferentes tanto de los viñamarinos 
$(M=1.2, D E=1.46)$, los nortinos $(M=0.4, D E=$ 1.79), y por último los santiaguinos $(M=-0.4, D E$ $=1.71$ ), en donde se presenta la única evaluación negativa $\left(M=0, t_{(80)}:-2.19, p=0.03\right)$.

Considerando la evaluación que hacen los dos grupos de edad (jóvenes y adultos) con relación a las características de los magallánicos podemos ver que los adultos tienen mejor percepción de si mismos $(M=2.4, D E=0.65)$ que los jóvenes $(M=$ $1.6, D E=1.17), t_{(79)}=-3,95, p=0,0001$.

Para con los chilotes observamos la misma dirección, los adultos presentan una mejor evaluación $(M=2.4, D E=1.09)$, de las características que los jóvenes $(M=1.9, D E=1.05), t_{(81)}=-2,39, p=0,02$

En el caso de los viñamarinos no se presentan diferencias, los adultos perciben a estos $(M=1.3, D E=1.36)$, igual que los jóvenes $(M=$ $1.2, D E=1.56), t_{(79)}=-0,52$, n.s.

Hacia los nortinos observamos que existe una mejor evaluación de los adultos $(M=0.9, D E=$ $1.85)$, que de los jóvenes $(M=-0.01, D E=1.62)$, $t_{(81)}=-0,52, p=0,02$.

Para los santiaguinos la evaluación realizada al igual que para los magallánicos nos muestra que existe mejor percepción de los adultos $(M=-0.3, D E$ $=1.68$ ), que de los jóvenes, hacia los santiaguinos ( $M$ $=-0.8, D E=1.66), t_{(80)}=-2,32, p=0,04$. Mientras los adultos muestran un promedio cercano al punto neutro $\left(M=0, t_{(40)}=-0.13\right.$, n.s. $)$ los jóvenes presentan un promedio de evaluación que sitúa su percepción de los santiaguinos en el polo negativo $\left(M=0, t_{(40)}\right.$ $=--3.07, p<0.004)$

\section{DISCUSIÓN Y CONCLUSIÓN}

El objetivo de esta investigación fue describir los estereotipos de diferentes grupos sociales desde la perspectiva de dos subgrupos etarios magallánicos(jóvenes y adultos). Se abordó la interrogante de si existe un estereotipo negativo hacia las personas provenientes del norte, y en qué sector geográfico, dentro de los grupos consultados es más fuerte, y si entre los adultos magallánicos y los jóvenes magallánicos existen diferencias en la forma como perciben a diferentes grupos nacionales (los chilotes, viñamarinos, nortinos y santiaguinos). Mediante la utilización de dos técnicas diferentes, se buscó tanto la evaluación de dichas categorías sociales como la generación del perfil típico de las características de cada grupo social. Nuestra hipótesis inicial planteaba que los adultos mostrarían la presencia de una evaluación menos positiva que los jóvenes de las categorías exogrupales. Esta hipótesis no encontró apoyo empírico alguno ya que tanto al evaluar al endogrupo como al exogrupo, los adultos mostraron puntajes menos negativos. Aunque la explicación inicial podría haber sido la polarización de un prejuicio hacia el exogrupo "santiaguino", el resultado que señala que el auto-estereotipo también es menos positivo nos induce a interpretar estos resultados como la manifestación de un efecto de la etapa vita. Los jóvenes, con un promedio de edad que los califica dentro de lo que ha sido denominado "adolescencia tardía", podrían simplemente estar expresando un rasgo de este proceso de consolidación de su personalidad adulta que es el desarrollo del pensamiento crítico (Mietzel, 2005)

Como consecuencia, los resultados la presente investigación se focalizaron principalmente en describir la evaluación que un grupo de magallánicos realiza tanto de su propio grupo como de los chilotes, viñamarinos, nortinos y santiaguinos. Los resultados principales indican que al evaluar el auto-estereotipo mediante diferencial semántico, este se presenta básicamente como una descripción de adjetivos positivos. Vemos que los magallánicos encuestados se ven a sí mismos de manera positiva; tres de las cinco categorías superan el 90\% de aprobación, siendo "bueno" la que mayor aceptación tiene. Este resultado es concordante con la existencia de una identidad social clásica que presenta el sesgo del endogrupal. $\mathrm{Al}$ evaluar el estereotipo del conjunto de exogrupos calificables como provenientes del norte geográfico, son los "chilotes" quienes también presentan un alto grado de aceptación. Este resultado puede tener diversas interpretaciones dentro de la cuales tenemos la presencia de identidades sociales múltiples, una parte importante de los habitantes de la región se encuentran vinculados a algún otro grupo social que inmigró en las recientes generaciones de ancestros. Es así, como tanto los "chilotes" como otros grupos tales como los "croatas", son parte de la identidad magallánica. Además, existe una relación armónica con la cultura chilota que se ha entrelazado con la magallánica. Nuestra investigación no recolectó información sobre estas múltiples pertenencias por lo que esta interpretación de la positividad del estereotipo chilote deberá ser confirmada en futuras 
investigaciones.

Otros exogrupos que presentan estereotipos con tendencia positiva son los "viñamarinos" que obtiene en todas las categorías más del 60\% de aprobación y los "nortinos" que salvo el continuo atrayente/repulsivo todas superan el 50\% de aprobación. En el caso de los "viñamarinos", es posible que la asociación principal a esta categoría social sea la playa y las vacaciones, lo que implica una connotación positiva intrínseca. A diferencia de las relaciones instrumentales que los magallánicos suelen establecer con Santiago (ir a un médico especialista, realizar trámites específicos, etc.), la ciudad de Viña del Mar es un destino frecuente de vacaciones y se le vincula con un festival de la canción de larga tradición nacional.

En el extremo opuesto se encuentran los "santiaguinos", donde en una sola categoría logra superar el 50\% de aceptación (agradable/ desagradable), mientras que en todas las demás este nivel de aceptación se sitúa bajo el 50\%. Aunque los promedios globales tienden a situarse en un punto neutro y no de alta negatividad, es tanto la categoría social con menor promedio de positividad como aquella que reúne, en la generación espontánea de características, el mayor número de adjetivos negativos (agresivos, delincuentes, extrovertidos, individualistas, estresados y trabajadores). Es posible concluir, que la expresión popular "la gente mala del norte" expresa una forma de prejuicio hacia las personas provenientes de la ciudad de Santiago. Las razones de este resultado no son parte de los objetivos del presente estudio pero bien podría expresar la presencia de una dependencia relativa entre la capital del país y la región que conflictúa lo cotidiano en áreas tan diversas como lo político y social.

Los resultados, como una medida de estereotipo, muestran las "creencias", imágenes mentales de un grupo de encuestados y no permiten pronosticar la existencia o desarrollo de prejuicio o, incluso acciones de discriminación hacia miembros de estos grupos. Como medida de actitud, representa una visión de la realidad que no necesariamente se traducirá en comportamientos. Como creencia compartida, de revelarse extensa, podría constituirse en una representación social que daría cuenta de las relaciones inter e intragrupales.

Una serie de interrogantes surgen a partir de estos resultados preliminares: ¿Son los santiaguinos sólo un chivo expiatorio para no reconocerse a sí mismos con pros y contras?, ¿Será un castigo inconsciente hacia el centralismo que ejerce la capital nacional, muchas veces repudiado por los magallánicos? ¿Por qué al parecer los jóvenes tienen una tendencia a evaluar menos positivamente?, etc.

Debido a que es un tema de carácter social, que toca las relaciones intergrupales, la investigación en este ámbito podría resultar en un aporte a cuestiones vinculadas con la identidad regional, sobre todo en el caso de evidenciarse la existencia de prejuicio utilizándose la figura del "nortino" como chivo expiatorio para la explicación de actividades relacionadas con el delito y/o comportamientos no deseados en la región.

Como limitante tenemos que la presente investigación es un estudio preliminar, realizado sobre un número reducido de personas habitantes de la ciudad de Punta Arenas. Interesante resultaría ampliar el estudio tanto en número como en amplitud geográfica regional con el objetivo de observar si este fenómeno se presenta de igual manera en otras ciudades, y si disminuye o aumenta. La necesidad de medir otras variables explicativas tales como el regionalismo o la identificación con lo regional, representa un desafío para estudios posteriores, así como también la evaluación directa o indirecta de la orientación hacia el comportamiento discriminatorio. Sería interesante que futuras investigaciones se orientaran a la exploración de estas interrogantes, pudiendo indagar en los efectos que estos estereotipos tienen en las relaciones interpersonales entre magallánico - santiaguino, encontrar que similitudes y diferencias ven los magallánicos hacia con los demás grupos, cómo se presenta la convivencia cotidiana, y de dónde surgen y cómo se mantienen estos estereotipos. La cuestión de las identidades sociales múltiples también es una cuestión pendiente e insoslayable en una categoría social construida por la inmigración tanto nacional como internacional. Esto permitiría profundizar la comprensión de alguno de los resultados tales como las similitudes observadas con el grupo denominado "chilote". Es un tema interesante de seguir profundizando, que genera aún hoy en día debates y creencias. 


\section{BIBLIOGRAFÍA}

Allen, B. y Potkay, C. (1973). Variability of self-description on a day-to-day basis: Longitudinal use of the adjective generation technique. Journal of Personality, 41(4), $638-652$

Allport, G. W. (1977). La Naturaleza del Prejuicio. Buenos Aires: Universitaria.

Anderson, B. (1993). Comunidades imaginarias. Reflexiones sobre el origen y la difusión del nacionalismo. México: Fondo de Cultura Económica.

Berkowitz, L. (1979). Advances in Experimental Social Psychology. New York: Academic Press.

Cruz, P. (2000). La autonomía que la Región de Magallanes necesita: Entrevista - Mateo Martinic, Premio Nacional de Historia 2000. Mundosur Magazine http://mundosur. com/magazine/politica.htm.

Facchinetti, G., Jensen, S., \& Zaffrani, T. (2000). Patagonia: Historia, discurso e imaginarios. Temuco: Ediciones Universidad de la Frontera.

Festinger, L. (1957). A theory of cognitive dissonance. Palo Alto, CA: Stanford University.

Franzoi, S. (2007). Psicología Social. México: McGrawHill.

Hamilton, D. L. (1979). A cognitive-attributional analysis of stereotyping. En L. Berkowitz (ed.). Advances in Experimental Social Psychology. Nueva York: Académic Press.

Hogg, M.A., \& Abrams, D. (1999). Social identity and social cognition. Malden: Blackwell Publishers.

Hogg, M. A., \& Turner, J. C. (1987). Social identity and conformity: A theory of referent informational influence. Cambridge: Cambridge University Press.

Klineberg, O. (1973). Psicología Social. México: Fondo de la Cultura Económica.
Krech, D. (1978). Psicología Social. Madrid: Biblioteca nueva. Lippmann, W. (1922). Public Opinion. New York: Macmillan.

Mackie, M. M. (1973). Arriving at truth by definition: Case of stereotype inaccuracy. Social Problems. California: University of California Press.

Martinic, M. (1992). Historia de la Región Magallánica. Punta Arenas: Universidad de Magallanes.

McCauley, C., Stitt, C. L., \& Segal, M. (1980). Stereotyping: From prejudice to prediction. En: Psychological Bulletin.

Mietzel, G. (2005). Clases de la Psicologia Evolutiva. Madrid: Agapea.

Morales, J. F., \& Moya, M.C. (1996). Tratado de Psicología Social. Volumen I: Procesos básicos. Madrid: Síntesis Psicología.

Morales, J.F., Páez, K D., Kornblit, A. L., \& Asún, D. (2001). Psicología Social. Buenos Aires: Prentice Hall.

Myers, D. (2000). Psicología Social. México: McGraw-Hill.

Osgood, C., Suci, G., \& Tannenbaum, P. (1976). El diferencial semántico como instrumento de medida. Buenos Aires: Nueva Visión.

Ross, L., G., Bierbrauer, G., \& Hoffman, S. (1976). The role of attribution processes in conformity and dissent. American Psychologist, 31, 148 - 157.

Secord, P., \& Beckman C. (1976). Psicología Social. México: McGraw-Hill.

Tajfel, H. (1972). La catégorisation sociale. Paris: Larousse.

Tajfel, H. (1978). Differentiation between social groups: Studies in the social psychology of intergroup relations. London: Academic Press.

Tajfel, H. (1984). Grupos Humanos y Categorías Sociales. Barcelona: Herder.

Worchel, S. (2004). Psicología Social. Madrid: Thomson. 
